\title{
SNAP: A Multi-Stage XML-Pipeline for Aspect Based Sentiment Analysis
}

\author{
Clemens Schulze Wettendorf and Robin Jegan and Allan Körner and Julia Zerche \\ and Nataliia Plotnikova and Julian Moreth and Tamara Schertl and Verena Obermeyer \\ and Susanne Streil and Tamara Willacker and Stefan Evert
}

\author{
Friedrich-Alexander-Universität Erlangen-Nürnberg \\ Department Germanistik und Komparatistik \\ Professur für Korpuslinguistik \\ Bismarckstr. 6, 91054 Erlangen, Germany

\begin{abstract}
\{clemens.schulze.wettendorf, robin.jegan, allan.koerner, julia.zerche, nataliia.plotnikova, julian.moreth, tamara.schertl, verena.obermeyer, susanne.streil, tamara.willacker, stefan.evert\}efau.de
\end{abstract}

\begin{abstract}
This paper describes the SNAP system, which participated in Task 4 of SemEval2014: Aspect Based Sentiment Analysis. We use an XML-based pipeline that combines several independent components to perform each subtask. Key resources used by the system are Bing Liu's sentiment lexicon, Stanford CoreNLP, RFTagger, several machine learning algorithms and WordNet. SNAP achieved satisfactory results in the evaluation, placing in the top half of the field for most subtasks.
\end{abstract}

\section{Introduction}

This paper describes the approach of the SemaNtic Analyis Project (SNAP) to Task 4 of SemEval2014: Aspect Based Sentiment Analysis (Pontiki et al., 2014). SNAP is a team of undergraduate students at the Corpus Linguistics Group, FAU Erlangen-Nürnberg, who carried out this work as part of a seminar in computational linguistics.

Task 4 was divided into the four subtasks $A s$ pect term extraction (1), Aspect term polarity (2), Aspect category detection (3) and Aspect category polarity (4), which were evaluated in two phases (A: subtasks 1/3; B: subtasks 2/4). Subtasks 1 and 3 were carried out on two different datasets, one of laptop reviews and one of restaurant reviews. Subtasks 2 and 4 only made use of the latter.

This work is licensed under a Creative Commons Attribution 4.0 International Licence. Page numbers and proceedings footer are added by the organisers. Licence details: http: //creativecommons.org/licenses/by/4.0/

\begin{tabular}{|c|c|c|c||c|}
\hline Task & Dataset & Rank & Score & Best \\
\hline 1 & Lap & 10 of 21 & $\mathbf{0 . 6 2 4}$ & 0.746 \\
1 & Res & 20 of 21 & $\mathbf{0 . 4 6 5}$ & 0.840 \\
3 & Res & 6 of 15 & $\mathbf{0 . 7 8 2}$ & 0.886 \\
\hline 2 & Lap & 7 of 23 & $\mathbf{0 . 6 4 1}$ & 0.705 \\
2 & Res & 12 of 24 & $\mathbf{0 . 7 0 8}$ & 0.810 \\
4 & Res & 11 of 18 & $\mathbf{0 . 6 9 6}$ & 0.829 \\
\hline
\end{tabular}

Table 1: Ranking among constrained systems.

The developed system consists of one module per subtask, in addition to a general infrastructure and preprocessing module, All modules accept training and test data in the XML format specfied by the task organizers. The modules can be combined into a pipeline, where each step adds new annotation corresponding to one of the four subtasks.

Table 1 shows our ranking among all constrained systems (counting only the best run from each team), the score achieved by SNAP (accuracy or F-score, depending on subtask), and the score achieved by the best system in the respective subtask. Because of a preprocessing mistake that was only discovered after phase A of the evaluation had ended, results for subtasks 1 and 3 are significantly lower than the results achieved during development of the system.

\section{Sentiment lexicon}

Early on in the project it was decided that a comprehensive, high-quality sentiment lexicon would play a crucial role in building a successful system. After a review of several existing lexica, Bing 
Liu's sentiment word list (Hu and Liu, 2004) was taken as a foundation and expanded with extensive manual additions.

The first step was an exhaustive manual websearch to find additional candidates for the lexicon. The candidates were converted to a common format, and redundant entries were discareded. The next step consisted of further expansion with the help of online thesauri, from which large number of synonyms and antonyms for existing entries were obtained. Since the coverage of the lexicon was still found to be insufficient, it was further complemented with entries from two other existing sentiment lexica, AFINN (Nielsen, 2011) and MPQA (Wilson et al., 2005).

Finally the augmented lexicon was compared with the original word lists from AFINN, MPQA and Bing Liu in order to measure the reliabilty of the entries. The reliability score of each entry is the number of sources in which it is found.

\section{Infrastructure and preprocessing}

Within the scope of Task 4 - but not one of the official subtasks - the goal of the infrastructure module was (i) to support the other modules with a set of project-specific tools and (ii) to provide a common API to the training and test data augmented with several layers of linguistic annotation. In order to roll out the required data as quick as possible, the Stanford CoreNLP suite ${ }^{1}$ was used as an off-the-shelf tool. The XML files provided by the task organizers were parsed with the xml.etree.ElementTree API, which is part of the standard library of Python 2.7.

Since the module for subtask 1 was pursuing an IOB-tagging approach for aspect term identification, the part-of-speech tags provided by CoreNLP had to be extended. During the process of merging the original XML files with the CoreNLP annotations, IOB tags were generated indicating whether each token is part of an aspect term or not. See Section 4 for further information.

For determining the polarity of an aspect term, the subtask 2 module made use of syntactic dependencies between words (see Section 5 for details). For this purpose, the dependency trees produced by CoreNLP were converted into a more accessible format with the help of the Python software package NetworkX. ${ }^{2}$.

\footnotetext{
${ }^{1}$ http://nlp.stanford.edu/software/corenlp.shtml

${ }^{2}$ http://networkx.github.io/
}

\section{Aspect term extraction}

The approach chosen by the aspect term extraction module (subtask 1) was to treat aspect term extraction as a tagging task. We used a standard IOB tagset indicating whether each token is at the beginning of an aspect term (ATB), inside an aspect term (ATI), or not part of an aspect term at all (ATX).

First experiments were carried out with unigram, bigram and trigram taggers implemented in NLTK (Bird et al., 2009), which were trained on IOB tags derived from the annotations in the Task 4 gold standard (comprising both trial and training data). We also tested higher-order n-gram taggers and the NLTK implementation of the Brill tagger (Brill, 1992).

For a more sophisticated approach we used RFTagger (Schmid and Laws, 2008), which extends the standard HMM tagging model with complex hidden states that consist of features corresponding to different pieces of information. RFTagger was developed for morphological tagging, where complex tags such as N.Reg.Nom.Sg. Neut are decomposed into the main syntactic category (N) and additional morpho-syntactic features representing case ( $\mathrm{Nom})$, number $(\mathrm{Sg})$, etc.

In our case, the tagger was used for joint annotation of part-of-speech tags and IOB tags for the aspect term boundaries, based on the rationale that the additional information encoded in the hidden states (compared to a simple IOB tagger) would allow RFTagger to learn more meaningful aspect term patterns. We decided to encode the IOB tags as the main category and the part-of-speech tags as additional features, since changing these categories, meaning POS tags as the main category and IOB tags as additional features, had resulted in lesser performance. The training data were thus converted into wordannotation pairs such as screen_AT/ATB.NN or beautiful/ATX. JJ. Note that known aspect terms from the gold standard (as well as additional candidates that were generated through comparisons of known aspect terms with lists from WordNet) were extended with the suffix _AT in a preprocessing step. Our intention was to enable the tagger to learn directly that tokens with this suffix are likely to be aspect terms.

Table 2 shows tagging accuracy for different algorithms, computed by ten-fold cross-validation over a gold standard comprising the training and 


\begin{tabular}{|l|l|l|}
\hline Tagger & Rest. & Laptops \\
\hline Unigram & $83.41 \%$ & $83.91 \%$ \\
Bigram (backoff: U) & $85.37 \%$ & $85.74 \%$ \\
Trigram (backoff: U+Bi) & $85.41 \%$ & $86.33 \%$ \\
Brill (backoff: U+Bi+T) & $85.48 \%$ & $86.47 \%$ \\
RFTagger & $\mathbf{9 5 . 2 0 \%}$ & $\mathbf{9 6 . 4 7 \%}$ \\
\hline
\end{tabular}

Table 2: Accuracy of different aspect term taggers.

trial data sets. The table shows that the bigram, trigram and Brill taggers achieve only marginal improvements over a simplistic unigram tagger, even when they are combined through back-off linking. The RFTagger achieved by far the best accuracy on both data sets.

\subsection{Results and debugging}

Our final results for the full aspect term extraction procedure are shown in Table 3.

\begin{tabular}{|l|l|l|}
\hline Score & Rest. & Laptops \\
\hline Precision & $57.14 \%$ & $64.54 \%$ \\
Recall & $39.15 \%$ & $60.40 \%$ \\
$\mathrm{~F}_{1}$-Score & $46.47 \%$ & $62.40 \%$ \\
\hline
\end{tabular}

Table 3: Aspect term extraction results.

The huge difference between tagging accuracy achieved in the development phase and the aspect term extraction quality obtained on the SemEval2014 test set is caused by different factors. First, Table 2 shows the tagging accuracy across all tokens, not limited to aspect terms. A tagger that works particularly well for many irrelevant tokens (punctuation, verbs, etc.), correctly marking them ATX, may achieve high accuracy even if it has low recall on tokens belonging to aspect terms. Second, the official scores only consider an aspect term candidate to be a true positive if it covers exactly the same tokens as the gold standard annotation. If the tagger disagrees with the human annotators on whether an adjective or determiner should be considered part of an aspect term, this will be counted as a mistake despite the overlap. Thus, even a relatively small number of tagging mistakes near aspect term boundaries will be punished severly in the evaluation. Unseen words as well as long or unusual noun phrases turned out to be particularly difficult.

Table 3 indicates a serious problem with the restaurant data, which has surprisingly low recall, resulting in an $\mathrm{F}_{1}$-score almost 16 percent points lower than for the laptop data. A careful examination of the trial, training and test data revealed an early mistake in the preprocessing code as the main culprit. Once this mistake was corrected, the recall score for restaurants was similar to the score for laptops.

\section{Aspect term polarity}

Subtask 2 is concerned with opinion sentences, i.e. sentences that contain one or more aspect terms and express subjective opinions about (some of) these aspects. Such opinions are expressed through opinion words; common opinion words with their corresponding confidence values (numeric values from 1 to 6 expressing the level of certainty that a word is positive or negative, $\mathrm{cf}$. Sec. 2) are collected in sentiment lexica.

The preprocessing stage in this subtask starts with a sentence segmentation step that uses the output of the Stanford CoreNLP parser. ${ }^{3}$ All dependencies map onto a directed graph representation where words of each sentence are nodes in the graph and grammatical relations are edge labels. All aspect terms (Sec. 2) are marked in each dependency graph. When processing such a graph we extract all positive and negative opinion words occurring in each sentence by comparing them with word lists contained in our sentiment lexica. A corresponding confidence value from lexica is assigned for each opinion word, the number of positive and negative aspect terms occurring in each sentence are counted and their confidence values are summed up. These values serve as features for supervised machine learning using algorithms implemented in scikit-learn (Pedregosa et al., 2011).

All opinion words that build a dependency with an aspect term are stored for each sentence. A dominant word of each dependency is stored as a governor, whereas a subordinate one is stored as a dependent. Both direct and indirect dependencies are processed. If there are several indirect dependencies to an aspect term, they are processed recursively. Using lists of extracted dependencies between opinion words and aspect terms handwriten rules assign corresponding confidence values to aspect terms.

\footnotetext{
${ }^{3}$ nlp.stanford.edu/software/dependencies_manual.pdf
} 


\subsection{Features based on a sentiment lexica}

The extended sentiment dictionaries were used to extract five features: I) tokens expressing a positive sentiment belonging to one aspect term, II) tokens expressing a negative sentiment, III) confidence values of positive tokens, IV) confidence values of negative tokens, V) a sum of all confidence values for all positive and all negative opinion words occurring in a sentence.

\subsection{Features based on hand-written rules}

We made use of direct and indirect negation markers, so that all opinion words belonging to a negated aspect term swap their polarity signs. We added rules for negative particles not and no that directly precede an opinion word, for adverbs barely, scarcely and too, for such constructions as could have been and wish in the subjunctive mood. After swapping polarity signs of opinion words, a general set of hand-written rules was applied to the graph dependencies. The rules follow the order of importance of dependencies scaling from least important up to most important. We placed the dependencies in the following order: acomp, advmod, nsubjpass, conj_and, amod, prep_of, prep_worth, prep_on, prep_in, nsubj, infmod, dobj, xcomp, rcmod, conj_or, appos. All dependencies can be grouped into three categories based on the direction of the polarity assignment. The first group (acomp, advmod, amod, rcmod, prep_in) includes dependencies where a governor of a dependency takes over polarity of a dependent if the latter is defined. The second group (infmod, conj_or, prep_on, prep_worth, prep_of, conj_and) covers dependencies in which a dependent element takes over polarity of a governor if the latter is defined. The third group (dobj, xcomp) is for cases when both governor and dependent are defined. Here a governor takes over polarity of a dependent.

\subsection{Experiments}

In this section we compare two approches to aspect term polarity detection. The first approach simply counts all positive and negative words in each sentence and then assigns a label based on which of the two counts is larger. It does not make use of machine learning techniques and its accuracy is only about $54 \%$. Results improve significantly with supervised machine learning based on the feature sets described above. We experimented with different classifiers (Maximum Entropy, Linear SVM and SVMs with RBF kernel) and various subsets of features. By default, we worked on the level of single opinion words that express a positive or negative polarity (sg). We added the following features in different combinations: an extended list of opinion words (ex) obtained from a distribution semantic model, based on nearest neighbours of known opinion words (Proisl et al., 2013); potential misspellings of know opinion words, within a maximal Levenshtein distance of 1 (lv); word combinations and fixed phrases (ml) containing up to 3 words (e.g., good mannered, put forth, tried and true, up and down); and the sums of positive and negative opinion words in the whole sentence (st). The best results for the laptops data were achieved with a Maximum Entropy classifier, excluding misspellings (lv) and word combinations (ml); the corresponding line in Table 4 is highlighted in bold font. Even though MaxEnt achieved the best results during development, we decided to use SVM with a RBF kernel for the test set, assuming that it would be able to exploit interdependencies between features. The accuracy achieved by the submitted system is highlighted in italics in the table. The training test data provided for restaurants and laptops categories were split equally into two sets where the first set (first half) was used for training a model and the second set was used for the test and evaluation stages. Experiments on the restaurants data produced similar results.

\begin{tabular}{|l|c|c|c|c|c|l|}
\hline classifier & sg & ex & lv & ml & st & Acc \\
\hline MaxEnt & + & + & - & - & - & 0.5589 \\
MaxEnt & + & + & + & - & - & 0.4905 \\
MaxEnt & + & + & - & + & - & 0.5479 \\
MaxEnt & + & + & - & - & + & $\mathbf{0 . 6 5 0 6}$ \\
MaxEnt & + & + & - & + & + & 0.5742 \\
SVM $_{\mathrm{rbf}}$ & + & + & - & - & - & 0.5581 \\
$\mathrm{SVM}_{\mathrm{rbf}}$ & + & + & + & - & - & 0.4905 \\
$\mathrm{SVM}_{\mathrm{rbf}}$ & + & + & - & + & - & 0.5479 \\
$\mathrm{SVM}_{\mathrm{rbf}}$ & + & + & - & - & + & 0.6402 \\
$\mathrm{SVM}_{\mathrm{rbf}}$ & + & + & - & + & + & 0.5717 \\
\hline
\end{tabular}

Table 4: Results for laptops category on train set.

\section{Aspect category detection}

Subtask 3 deals with determining which aspect categories out of a predefined set occur in a given sentence. The developed module consists of two 
independent parts - one based on machine learning, the other on similarities between WordNet synsets ("synonym sets", roughly corresponding to concepts). While both approaches achieved similar performance during development, combining them resulted in overall better scores. However, the success of this method crucially depends on accurate indentification of aspect terms.

\subsection{A WordNet-based approach ${ }^{4}$}

The WordNet-based component operates on previously identified aspect terms (from the gold standard in the evaluation, but from the module described in Sec. 4 in a real application setting). For each term, it finds all synsets and compares them to a list of "key synsets" that characterize the different aspect categories (e.g. the category food is characterized by the key synset meal.n.01, among others). The best match is chosen and added to an internal lexicon, which maps each unique phrase appearing as an aspect term to exactly one aspect category. As a similarity measure for synsets we used path similarity, which determines the length of the shortest path between two synsets in the WordNet hypernym/hyponym taxonomy. Key synsets were extracted from a list of high frequency terms and tested manually to create an accurate represenation for each category.

In the combined approach this component was taken as a foundation and was augmented by highconfidence suggestions from the machine learning component (see below).

Additional extensions include a highconfidence lexicon based on nearest neighbours from a distributional semantic model, a rudimentary lexicon of international dishes, and the application of a spellchecker; together, they accounted only for a small increase in F-score on the development data (from 0.758 to 0.768 ).

\subsection{A machine learning approach $^{5}$}

The machine learning component is essentially a basic bag-of-words model. It employs a multinomial Naive Bayes classifier in a one-vs-all setup to achieve multi-label classification. In addition to tuning of the smoothing parameters, a probability threshold was introduced that every predicted category has to pass to be assigned to a sentence.

\footnotetext{
${ }^{4}$ We used the version of WordNet included in NLTK 2.0.4 (Bird et al., 2009), accessed through the NLTK API.

${ }^{5}$ We used machine learning algorithms implemented in scikit-learn 0.14.1 (Pedregosa et al., 2011).
}

\begin{tabular}{|l|l|l|l||c|}
\hline Test & Train & AT & Mode & F $_{1}$ \\
\hline SE14 & Dev & Sub1 & All & 0.782 \\
SE14 & Dev* & Sub1 & WN & 0.666 \\
SE14 & Dev & Sub1* & ML & $\mathbf{0 . 7 8 8}$ \\
\hline SE14 & Dev & Gold & All & $\mathbf{0 . 8 4 8}$ \\
SE14 & Dev* & Gold & WN & 0.829 \\
SE14 & Dev & Gold* & ML & 0.788 \\
\hline Dev (cv) & Dev & Gold & All & $\mathbf{0 . 8 0 0}$ \\
Dev (cv) & Dev* & Gold & WN & 0.768 \\
Dev (cv) & Dev & Gold* & ML & 0.769 \\
\hline
\end{tabular}

*indicates data sets not used by a given component

Table 5: Aspect category detection results.

Different thresholds were used for the stand-alone component $(t h=0.625)$ and the combined approach $(t h=0.9)$. In the latter case all predicitions of the WordNet-based component were accepted, but only high-confidence predictions from the Naive Bayes classifier were added.

\subsection{Results}

Table 5 summarizes the results of different experiments with aspect category detection. In all cases the training data consisted of the combined official train and trial sets (Dev). The last three rows show results obtained by ten-fold cross-validation in the development phase, the other rows show the corresponding results on the official test set (SE14). The first three rows are based on automatically detected aspect terms from the module described in Sec. 4 (Sub 1), the other rows used gold standard aspect terms. Separate results are provided for the combined approach (Mode: All) as well as for the two individual components $(\mathrm{WN}=$ WordNet, $\mathrm{ML}$ = machine learning). Note that the WN component does not require any training data, while the ML component does not make use of aspect terms marked in the input.

With gold standard aspect terms, the WordNetbased approach is equal to or better than the Naive Bayes classifier, and best results are achived by a combination of the two components. However, the poor accuracy of the automatic aspect term extraction (cf. Table 3) has a disastrous effect: even the combined approach used in the official submission performs less well than the ML component alone. Nevertheless the experiment with gold standard aspect terms suggests that the matching from aspect term to category works quite well, with a small additional improvement from the Naive 
Bayes bag-of-words model.

\section{Aspect category polarity}

The general approach was to allocate each aspect term to the corresponding aspect categories. A simple rule set was then used to determine the polarity of each aspect category based on the polarities of the aligned aspect terms. In cases where no aspect terms are marked (but sentences are still labelled with aspect categories), the idea was to fall back on the sentiment values for the entire sentences provided by the CoreNLP suite. ${ }^{6}$

\subsection{Term / category alignment}

To establish a basis for creating the mapping rules, the first step was to work out the distribution of aspect terms and aspect categories in the training data. The most common case is that an aspect category aligns with a single aspect term $(1476 \times)$; there are also many aspect categories with multiple aspect terms $(1179 \times)$ and some aspect categories without any aspect terms. Since the WordNet-Approach from Sec. 6 showed relatively good results (especially if gold standard aspect terms are available, which is the case here), a modified version was used to assign each aspect term to one of the annotated categories.

\subsection{Polarity allocation}

After the assignment of aspect terms to their according aspect category - if needed - the aspect category polarity can be determined. For this, the polarity values of all aspect terms assigned to this category were collected, and duplicates were removed in order to produce a unique set (e.g. 1, $1,-1,0,0$ would be reduced to $1,-1,0)$. A set with both negative and positive polarity values indicates a conflict for the corresponding aspect category, while a neutral polarity value would be ignored, if positive or negative polarity values occur. Our method achieved an accuracy of $89.16 \%$ for sentences annotated with just a single aspect category. In cases where only one aspect term had been assigned to a aspect category the accuracy was unsuprisingly high $(96.61 \%)$, whereas the accuracy decreased in cases of multiple assigned aspect terms $(78.44 \%)$. For aspect categories without aligned aspect terms, as well as the category anecdotes/miscellaneous, the sentiment values of the CoreNLP sentiment analysis tool had to be

\footnotetext{
${ }^{6}$ http://nlp.stanford.edu/software/corenlp.shtml
}

used, which led to a poor accuracy in those cases, namely $52.74 \%$.

\subsection{Results}

On the official test set, the module for subtask 4 achieved an accuracy of $69.56 \%$. An important factor is the very low accuracy in cases where the CoreNLP sentiment value for the entire sentence had to be used. We expect a considerable improvement from using a modified version of the subtask 2 module (Sec. 5) to compute overall sentence polarity.

\section{Conclusion}

We have shown a modular system working as a pipeline that modifies the input sentences step by step by adding new information as XML tags. Aspect term extraction was handled as a tagging task that utilized an IOB tagset to find aspect terms with the final version relying on Schmid's RFTagger. Determination of aspect term polarity was achieved through a machine learning approach that uses SVMs with RBF kernel. This was supported by an augmented sentiment lexicon based on several different sources, which was expanded manually by a team of students. Aspect category detection in turn employs a combination approach of an algorithm depending on WordNet synsets and a bag-of-words Naive Bayes classifier. Finally aspect category polarity was calculated by combining the results from the last two modules.

Overall results were satisfactory, being mostly in the top half of submitted systems. During phase A of testing (subtasks 1 and 3), a preprocessing error caused a massive drop in performance in aspect term extraction. This carried over to the other subtask, because the module uses aspect terms among other features to identify aspect categories. Scores for phase B (subtasks 2 and 4) were very close to test results during development with the exception of cases where the CoreNLP sentiment value for an entire sentence had to be used.

\section{References}

Steven Bird, Ewan Klein, and Edward Loper. 2009. Natural Language Processing with Python. O'Reilly Media.

Eric Brill. 1992. A simple rule-based part of speech tagger. In Proceedings of the Third Conference on Applied Natural Language Processing, pages 152155 , Trento, Italy. 
Minqing $\mathrm{Hu}$ and Bing Liu. 2004. Mining and summarizing customer reviews. In Proceedings of the 10th ACM SIGKDD International Conference on Knowledge Discovery and Data Mining (KDD '04), pages 168-177, Seattle, WA.

Finn Årup Nielsen. 2011. A new ANEW: Evaluation of a word list for sentiment analysis in microblogs. In Proceedings of the ESWC2011 Workshop on Making Sense of Microposts: Big things come in small packages, number 718 in CEUR Workshop Proceedings, pages 93-98, Heraklion, Greece, May.

Fabian Pedregosa, Gaël Varoquaux, Alexandre Gramfort, Vincent Michel, Bertrand Thirion, Olivier Grisel, Mathieu Blondel, Peter Prettenhofer, Ron Weiss, Vincent Dubourg, Jake Vanderplas, Alexandre Passos, David Cournapeau, Matthieu Brucher, Matthieu Perrot, and Édouard Duchesnay. 2011. Scikit-learn: Machine learning in Python. Journal of Machine Learning Research, 12:2825-2830.

Maria Pontiki, Dimitrios Galanis, John Pavlopoulos, Harris Papageorgiou, Ion Androutsopoulos, and Suresh Manandhar. 2014. SemEval-2014 task 4: Aspect based sentiment analysis. In Proceedings of the 8th International Workshop on Semantic Evaluation (SemEval 2014), Dublin, Ireland.

Thomas Proisl, Paul Greiner, Stefan Evert, and Besim Kabashi. 2013. KLUE: Simple and robust methods for polarity classification. In Second Joint Conference on Lexical and Computational Semantics (*SEM), Volume 2: Proceedings of the Seventh International Workshop on Semantic Evaluation (SemEval 2013), pages 395-401, Atlanta, Georgia, USA, June. Association for Computational Linguistics.

Helmut Schmid and Florian Laws. 2008. Estimation of conditional probabilities with decision trees and an application to fine-grained POS tagging. In Proceedings of the 22nd International Conference on Computational Linguistics, volume 1, pages $777-$ 784.

Theresa Wilson, Janyce Wiebe, and Paul Hoffmann. 2005. Recognizing contextual polarity in phraselevel sentiment analysis. In Proceedings of the $\mathrm{Hu}$ man Language Technology Conference and Conference on Empirical Methods in Natural Language Processing (HLT-EMNLP 2005), pages 347-354, Vancouver, BC, Canada. 